\title{
Clinical Variability in Familial X-Linked Hypohidrotic Ectodermal Dysplasia with 2q12Mutation: A Rare Case Report of Female Siblings Agony
}

\author{
Nidhi Chhabra $^{1 *}$, Anuj Chhabra ${ }^{2}$ and Ruchi Mehta ${ }^{3}$ \\ ${ }^{1}$ M.D.S (Pedodontics), Senior Resident, Department of Dental Surgery, North DMC Medical College and Hindu Rao Hospital, Delhi, India. \\ ${ }^{2}$ M.D.S(Prosthodontics), Assistant Professor, Department of Dental Surgery, North DMC Medical College and Hindu Rao Hospital, Delhi, India.
}

${ }^{3}$ M.D.S (Oral Medicine, Diagnosis and Radiology), Reader, Department of Oral Medicine, Diagnosis and Radiology, Himachal Institute of Dental Sciences,

Paonta Sahib, Himachal Pradesh.

Received: September 13, 2017; Accepted: September 20, 2017 Published: October 09, 2017

*Corresponding author: Dr. Nidhi Chhabra, MDS Pedodontics, Senior Resident, Department of Dental Surgery, North DMC Medical College and Hindu Rao Hospital, Delhi, India, Tel: 9811175226;E-mail: nidhimahajandr84@gmail.com

\begin{abstract}
Background: Hypohidrotic Ectodermal dysplasia (HED) is a hereditary entity characterized by the abnormal development of embryonic ectodermally derived organs including sweat glands, teeth, and hair. The most prevalent form of HED is X-linked recessive with the gene mapping to Xq12-q13, seen mainly in males.
\end{abstract}

Case Report: Here, we present a rare case of autosomal Ectodermal dysplasia in two female siblings associated with defects of the $2 q 12$ region in both of their cell lines. Both siblings had variable alopecia, anhidrosis, molar hyperplasia and hypodontia. This paper highlights their oral rehabilitation, and the effects that the operative techniques had on their appearance and psyche.

Conclusion: The treatment resulted in excellent acclimatization to the prosthesis, improvement in the mastication, speech, and social attitude; thereby improving the quality of life.

Key words : Hypohidrotic Ectodermal dysplasia; anodontia; hypodontia

\section{Introduction}

Ectodermal dysplasia is a heterogeneous hereditary disorder characterized by aplasia or dysplasia of tissues of ectodermal origin, such as skin, hair, teeth, and sweat glands and nails [1] Ectodermal dysplasia syndrome was first described by Thurman in 1848 [2]. Although about 170 different subtypes of Ectodermal dysplasia have been recognized, these disorders are considered to be relatively rare with an estimated incidence of 1 case per 100,000 [3].

The most common, classic type is hypohidrotic ectodermal dysplasia, also known as Christ-Siemens-Touraine syndrome. Hypohidrotic Ectodermal dysplasia (HED) is most commonly inherited in an X-linked recessive pattern with the gene mapping to Xq12-q13, thus seen mainly in males; although rare cases of autosomal recessive and autosomal dominant inheritance have been identified. Hydrotic type however shows autosomal dominant inheritance pattern [4].

We report here a case of two female siblings with autosomal dominant HED presenting with alopecia, anhidrosis, hypodontia and molar hyperplasia. This paper highlights their management, and the effects that the operative techniques had on their appearance and psyche.

\section{Case Report}

Two siblings aged five years and two years were referred to our dental department because of delayed eruption of their primary teeth and the conical shape of the erupted teeth, which gave an unaesthetic facial appearance

\section{Medical and family history}

Both her parents were normal and had no history of such a condition. They had a consanguineous marriage. Familial history revealed that close relatives, including grandmother on the mother's side and cousins were afflicted with the same condition. The medical history was otherwise unremarkable. The pregnancies of the mother were described as normal, and no perinatal complications were mentioned. The mother gave a history of decreased sweating and their intolerance to heat, since childhood.

\section{Extra oral examination}

Both sisters presented with similar extraoral features, like the hair over the scalp were blond, fine, stiff, sparse, and short, while the eyebrows and eyelashes were missing. The skin was smooth, soft, dry, wrinkled especially around eyes and appeared prematurely aged. Fine linear wrinkles and increased 
pigmentation was present around the eyes and mouth. There was hyperkeratosis of the palms of the hands and soles of the feet. The face looked smaller because of frontal bossing and depression of the nasal bridge frontal bossing. Other features included a saddle-shaped nose, prominent supraorbital ridges and chin, protuberant lips, and a reduced vertical dimension of the lower face, which gave them an aged appearance (Figure 1). Systemic examination was normal.

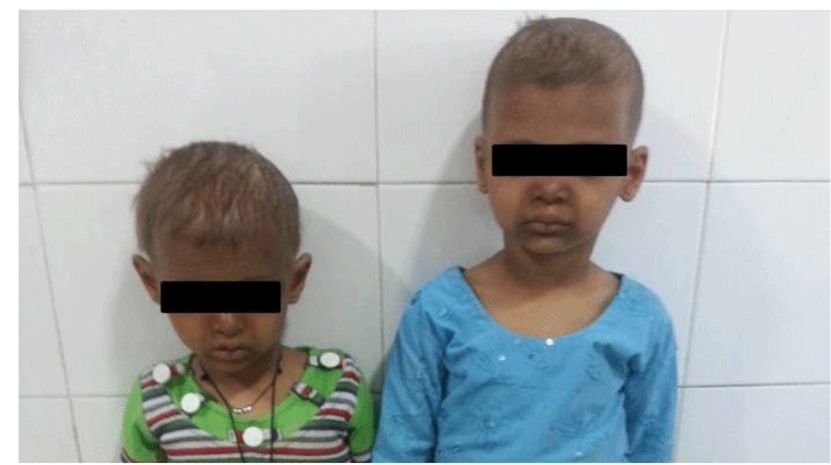

Figure 1: Pre-treatment profile photograph of the siblings aged 5 years and 2 years, with the characteristic facial appearance, including a prominent forehead, saddle-shaped nose, prominent lips, increased pigmentation around the eyes and mouth, sparse, dry scalp hair, eyelashes, and eyebrows

\section{Extra oral examination}

An oral examination revealed that only two of their 20 primary teeth were present; they were the maxillary right and left central incisors, both of which were markedly tapered. Thin alveolar crests were present (Figure 2 and 3). The problems associated with partial anodontia were the difficulty in chewing and unesthetic appearance.

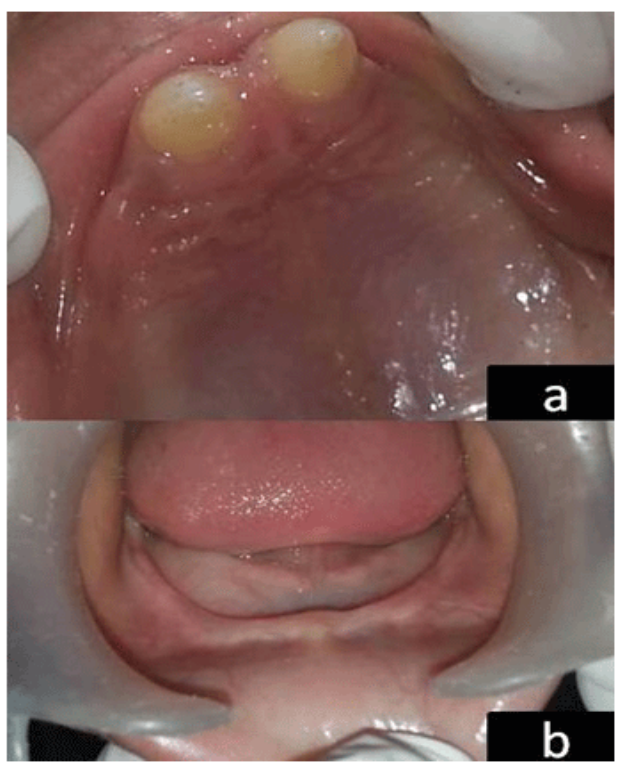

Figure 2: PIntraoral view of the 5 year old patient (a) Maxillary arch showed the presence of 2 conical teeth (b) Edentulous mandibular ridge

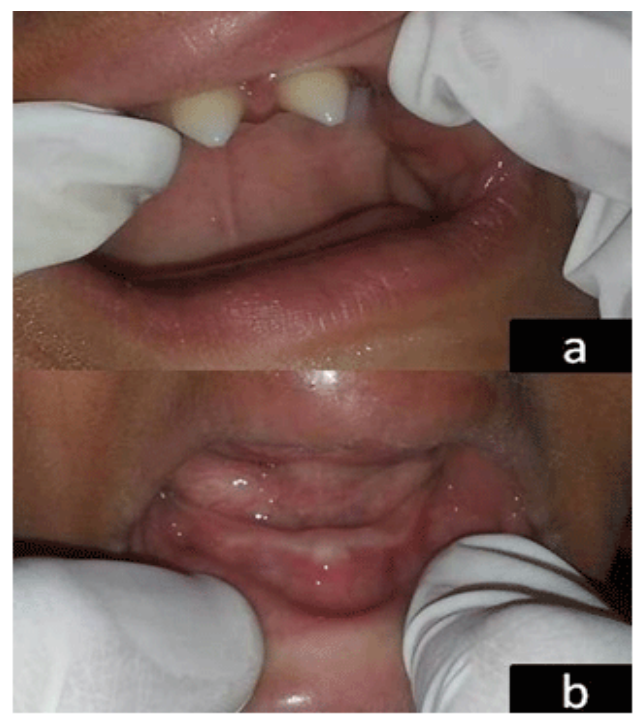

Figure 3: Intraoral view of the 3 year old sibling (a) Maxillary arch showed the presence of 2 conical teeth (b) Edentulous mandibular ridge

\section{Radiographic examination}

A panoramic radiograph revealed the absence of all primary and permanent teeth except maxillary central primary and permanent incisors (Fig 4A and B).

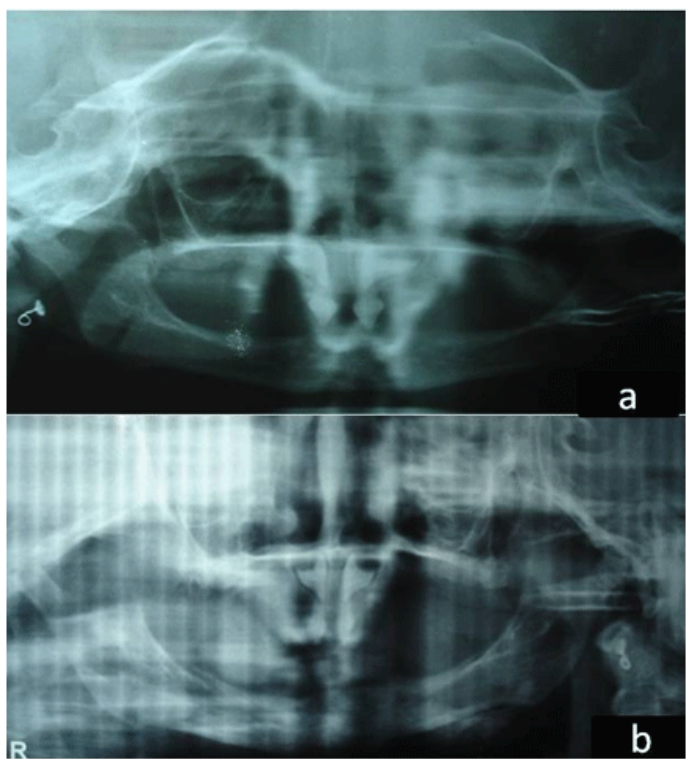

Figure 4: Panoramic radiographic of two cases of Hypohidrotic Ectodermal dysplasia. Panoramic radiograph of the (a) 5 year old patient and (b) of the second girl (3 year old). The congenital absence of the majority of teeth is apparent. In the first case, only two teeth are present in the maxilla. In the second, only two tooth are developing in the maxillary anterior region

Chromosomal analysis revealed a defect of the $2 \mathrm{q} 12$ region in both of their cell lines. Skin biopsies showed absence of apocrine glands in the axillary region and a comparative diminution of eccrine glands throughout. Consequently, based on the patient's 
history, clinical, radiographic and chromosomal examination, both siblings were diagnosed as suffering from rare case of autosomal dominant Hypohidrotic Ectodermal dysplasia. The treatment procedures were explained to the patients and their parents, and a written informed consent was obtained from them.

\section{Oral Rehabilitation Procedure}

The primary concern about the treatment of elder sibling was to meet the aesthetic and nutritional needs of the child. After the treatment, plan was explained to the parents, primary diagnostic impressions were made with irreversible hydrocolloid material (3M ESPE) mixed to a higher viscosity, to prepare casts for a diagnostic setup. These impressions were extremely difficult to take because of her initial uncooperative behaviour. Patient was not willing for extraction of deciduous teeth, so it was planned to retain the upper deciduous teeth and fabricate an overdenture. Custom trays were fabricated to eventually help make a superior final functional impression. Polyvinyl siloxane (Aquasil Soft Putty - Regular Set and Aquasil_LV Type III Light Bodied consistency, Dentsply) was used for border molding and as an impression material because of the required accuracy, better working time, and a fast setting time. Occlusal rims were fabricated chair side to determine the appropriate occlusal vertical dimension (OVD). Occlusal plane was confirmed using the fox plane, and the silicone-based bite registration material was utilized to record the centric jaw relation. Paediatric mold denture teeth were selected to replicate the natural dentition of a 5-year-old child. The trial bases were tried in the patient's mouth and confirmation of esthetics, OVD and centric relation records was done. The patient and her parents evaluated and approved the try-in arrangement of the teeth.

Heat-processed record bases were used to obtaining accurate jaw relation records for the over-denture fabrication as they provide superior stability, confirmation of the final retention, and easier adjustment of occlusion performed in the Iaboratory. The dentures were fabricated in heat-processed acrylic resin, finished, polished, inserted and pressure spots were adjusted(Fig 5). Post-insertion instructions were given to the patient and her mother; and they were educated about the maintenance and follow-up of the prosthesis. The mother was told to use $0.4 \%$ topical stannous fluoride on the remaining two maxillary anterior teeth, employing the denture as a fluoride carrier.

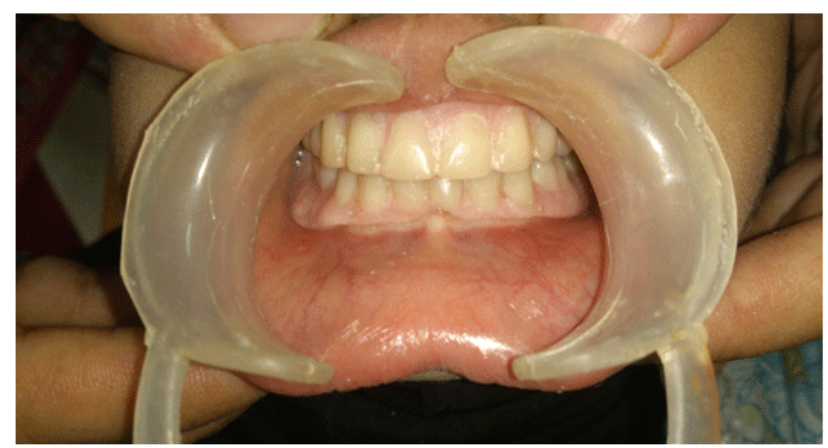

Figure 5: Over denture inserted in maxillary arch and complete denture in mandibular arch
Regular recalls were scheduled for 24-hour, 1-week, and 3-month intervals to make any needed adjustments, because of oral hygiene issues and dental caries risk of abutment teeth. The patient and her mother were pleased over the outcome of the prosthesis. Further treatment will include modifications of the dentures by relining or replacement as per the need of skeletal growth.

Because of the management problems and a severe gag reflex in younger sibling, dental treatment could not be performed. Arrangements were made to review her every four months until she could cooperate sufficiently to receive dental treatment.

\section{Discussion}

The most prevalent type of Ectodermal dysplasia is the anhidrotic/Hypohidrotic type illustrating a triad of dentition anomalies, partial or complete absence of the sweat glands, and hypotrichosis. Most cases of HED are X-linked recessive associated with mutations on the EDA gene [5]. However, in our rare case two female siblings of autosomal Ectodermal dysplasia presented with defects of the $2 \mathrm{q} 12$ region in both of their cell lines.

The typical faces of HED are characterized by frontal bossing, malar/midfacial hyperplasia, sparse, hypo pigmented hair, a saddle nose, thick everted lips, prominent supraorbital ridges, hyperkeratosis of the palms of the hands and soles of the feet; abnormal pigmentation, wrinkles around the eyes; which were seen in both the siblings $[6,7]$.

The oral phenotype includes multiple congenitally missing teeth, root and crown dysmorphism, mainly conical- shaped crowns, and reduced saliva flow; which was consistent in this case. Hypodontia or anodontia is the most common oral feature, illustrating the suppression of dental ectoderm. Alveolar bone is hypoplastic due to the absence of teeth, resulting in a loss of vertical dimension and protuberant lips [4]. The eruption of both dentitions may be delayed, or the teeth may remain unerupted, which was seen in our case.

Oral rehabilitation of children with Ectodermal dysplasia is mandatory to improve esthetics, speech, and masticatory efficiency as well as sagital and vertical skeletal relationships during craniofacial growth and development [8]. Early prosthetic treatment is usually suggested from 5 years of age. However, if the child is cooperative prosthetic intervention can be done in a patient as young as 2 or 3 years [9].

Prosthetic rehabilitation of such children is quite challenging and is further complicated by the growth and development of the child, variation in tooth development and eruption, the type of age-appropriate prosthesis, and the planning and timing of treatment [10]. Treatment options include a removable and/ or fixed partial denture, an overdenture, complete denture prosthesis or replacement of teeth by implants [11]. The implant retained prosthesis is usually limited to patients with completed craniofacial growth.

Although removable partial or complete dentures provide a simple, inexpensive and reversible Prosthodontics option, 
these require regular adjustments and should be replaced when a decreased vertical dimension of conclusion, and an abnormal mandibular posture are detected as per the need of craniofacial growth [12].

\section{Conclusion}

The treatment option preferred in our case was of an overdenture considering her age and her reluctance for extraction of the deciduous teeth. The atypical conical anterior teeth were used as abutments for overdenture In this situation, special consideration was paid to the impression making technique. The occlusion of the prosthesis was in harmony with the patient's occlusion. The patient's mother reported her excellent acclimatization to the prosthesis and improvements in her mastication, speech, and social attitude. The parents were educated about the future possibilities for dental implant placement, with the eventual goal of obtaining implant supported prosthesis.

\section{References}

1. Neville B, Damm D, Allen C, Bouquot J. Oral and Maxillofacial Pathology. $3^{\text {rd }}$ ed. Philadelphia: W.B. Saunders; 2008.

2. Yenisey M, Guler A, and Unal U. Orthodontic and prosthodontic treatment of ectodermal dysplasia: a case report. Br Dent J. 2004;196(11):677-679.

3. Vergo TJ Jr. Prosthodontics for pediatric patients with congenital/ developmental orofacial anomalies: A long-term follow-up. J Prosthet Dent. 2001;86(4):342-347.

4. Hekmatfar S, Jafari K, Meshki R, and Badakhsh S. Dental management of ectodermal dysplasia: two clinical case reports. J Dent Res Dent Clin Dent Prospect. 2012;6(3):108-112.
5. Kere J, Srivastava AK, Montonen 0, Zonana J, Thomas N, Ferguson $\mathrm{B}$, et al. X-linked anhidrotic (Hypohidrotic) ectodermal dysplasia is caused by mutation in a novel transmembrane protein. Nat Genet. 1996;13(4):409-416

6. de Aquino SN, Paranaiba LM, Swerts MS, Martelli DR, de Barros LM, Martelli Junior H. Orofacial Features of Hypohidrotic Ectodermal Dysplasia. Head and Neck Pathol. 2012;6(4):460-466.

7. Hickey AJ, Vergo TJ Jr. Prosthetic treatments for patients with ectodermal dysplasia. J Prosthet Dent. 2001;86(4):364-368.

8. Tarjan I, Gabris K, Rozsa N. Early prosthetic treatment of patients with ectodermal dysplasia: a clinical report. J Prosthet Dent. 2005;93(5):419-424.

9. Hickey AJ, Vergo TJ Jr. Prosthetic treatments for patients with ectodermal dysplasia. J Prosthet Dent. 2001;86(4):364-368.

10. C Bal, BT Bal, D Tufekcioglu. Treatment Considerations for a Patient with Hypohidrotic Ectodermal Dysplasia: A Case Report. J Contemp Dent Pract. 2008;9(3):1-7.

11. Kearns G, Sharma A, Perrott D, Schmidt B, Kaban L, Vargervik K. Placement of endosseous implants in children and adolescents with hereditary ectodermal dysplasia. Oral Surg Oral Med Oral Pathol Oral Radiol Endod.1999;88(1):5-10.

12. Franchi L, Branchi R, Tollaro I. Craniofacial changes following early prosthetic treatment in a case of hypohidrotic ectodermal dysplasia with complete anodontia. ASDC J Dent Child. 1998;65(2):116-121.

13. O'Dwyer MR, Renner RP, Fergusen FS. Overdenture treatment-one aspect of the team approach for the EEC syndrome patient. J Pedod. 1984;8:192-205. 